\title{
Railroading at the FDA
}

\author{
The US Food and Drug Administration approved a muscular-dystrophy drug against the scientific advice of its own \\ staff and advisors. Despite leadership's attempts to downplay the controversy, doubts now surround standards for \\ accelerated approval.
}

$\mathrm{O}_{\mathrm{g}}^{\mathrm{n}}$ 19 September, the US Food and Drug Administration (FDA) granted accelerated approval to Exondys 51 (Exondys, or eteplirsen), an exon-skipping antisense drug developed by Sarepta Therapeutics for the treatment of Duchenne muscular dystrophy (DMD), a progressive, ultimately lethal disease that primarily afflicts boys and has no other approved treatments. Although the decision received praise from patient groups, clinicians and Sarepta investors, it was based on what many consider to be inadequate scientific data. In the words of one FDA committee member, Exondys lowers the agency's evidentiary standard for drug effectiveness "to an unprecedented nadir."

FDA Commissioner Robert Califf has downplayed the spat in a memorandum as "the usual types of disagreement that occur when experts review clinical evidence." But a closer look at the FDA's internal memos reveals an approval process that was anything but usual.

The turmoil began in April, when Janet Woodcock, director of the Center for Drug Evaluation and Research, took the unusual step of cautioning those present at the Exondys advisory committee meeting of the "extreme" consequences of "failing to approve a drug that actually works" in devastating diseases. During the meeting, committee members found themselves "under intense and near-incessant pressure from a large public audience" packed with patients and advocates, all but one of whom gave testimony in favor of approval. Even so, the committee voted 7-3 (with three abstentions) against full approval and 7-6 against accelerated approval. Key concerns related to poor-quality data on the levels of dystrophin protein (the surrogate biomarker for drug efficacy), the use of historical controls and the lack of a correlation between clinical benefit, drug dosage and dystrophin abundance.

Following the negative vote, the FDA's Office of New Drugs (OND) recommended a rejection via "complete response" letter. But on 3 June, Woodcock instead offered Sarepta another lifeline, requesting that immunohistochemistry and western blots be performed on muscle biopsies from boys participating in an ongoing trial. Although the new data showed a nominal increase in dystrophin expression after drug administration, the gain was only to $0.22-0.30 \%$ above baseline. Whether such an increase is reasonably likely to predict clinical benefit became the basis of the unusual subsequent scientific dispute within the FDA.

Despite the underwhelming data, Woodcock issued an internal memo in July that recommended granting accelerated approval to Exondys. She chose not to wait for input from her review team, which, despite the new data, still recommended rejection.

The OND's Ellis Unger then took the extraordinary step of filing a formal complaint that alleged that Woodcock had inappropriately interfered with the scientific review process. After reviewing the dispute, the FDA's acting chief scientist, Luciana Borio, concluded that Woodcock's involvement "upended the typical review and decision-making process" and that "this could be the first time a Center Director has overruled a review team (and an advisory committee) on a question of whether effectiveness has been demonstrated." Stating that, in her view, the evidence was insufficient to support accelerated approval of Exondys, Borio recommended that Califf conduct a new scientific review of the drug application.

But in a 13-page memo, Califf deferred to Woodcock. "Overruling the Center Director is exceedingly rare," he noted, adding that this does "not set a general precedent for drug approvals under the accelerated approval pathway." Three days later, FDA granted Exondys accelerated approval and instructed Sarepta to conduct a two-year, randomized controlled trial to verify efficacy. Two members of the FDA review team resigned.

Despite Califf's demurral, this decision sends a signal that evidence from poorly designed clinical trials-if presented in the context of vocal patient advocacy - can be sufficient to obtain accelerated approval of a rare-disease drug. The inadequacy of the Exondys data is of particular concern because a 2013 publication (Ann. Neurol. 74, 637-647) funded by Sarepta suggested that Exondys could upregulate dystrophin by an unprecedented $52 \%$. This raised unreasonable expectations among patients with DMD, and although the authors stand by the study, Califf himself now says that it should be retracted in light of the FDA's re-analysis of the data.

Crucially, because commercial drug development is focusing increasingly on rare diseases, the factors that complicated the Exondys decisionvery small clinical trials, desperate patients lacking therapeutic options and reliance on surrogate endpoints-will come up again and again. With each rare-disease drug application, these challenges will be confronted anew, and they will risk more internal FDA disputes and staff resignations. The Exondys approval might also hamper the development of other drugs for DMD that are more effective; with Exondys on the market, it will be harder to persuade patients to enter not only Sarepta's postmarket trials, but also trials of any other drugs to treat DMD.

One way to accelerate and broaden data accrual on Exondys would be for the FDA to require the company to conduct a risk evaluation and mitigation strategy (REMS) directing DMD specialists to monitor and record findings from all patients who receive Exondys in the follow-up trial. In addition to providing information about safety and efficacy, this could reveal biomarkers that are predictive of response. Indeed, why not consider such an approach more broadly in the context of future accelerated approvals of drugs with limited efficacy data?

Everyone hopes that Exondys will prove effective as a treatment for DMD. In the meantime, insurance agency Anthem has refused to provide coverage. If, ultimately, Exondys is shown to lack efficacy, then the FDA must not hesitate to pull the drug. But it is likely that patients with DMD will fight the FDA vociferously over withdrawal.

Exondys's approval is a stark illustration of the challenges of balancing science with patient input in regulatory decisions. Insisting that this case is a one-off will not stop the aftershocks from reverberating throughout the biopharmaceutical industry. 